\title{
Structure genomics of two chimera plasmids p675920-1 and p675920-2 coexisting in a multi-drug resistant Klebsiella pneumoniae isolate
}

\author{
Jiao Feng ${ }^{1, *}$, Zhe Yin ${ }^{1,}{ }^{*}$, Zhe Zhan ${ }^{1}$, Haifeng Mao $^{2}$, Xiaoyuan Jiang ${ }^{1}$, Lijun Zeng ${ }^{1}$, \\ Wenhui Yang ${ }^{1}$, Huiying Yang ${ }^{1}$, Jinglin Wang ${ }^{1}$, Haijian Zhou ${ }^{3,4}$ and Dongsheng Zhou ${ }^{1}$ \\ ${ }^{1}$ State Key Laboratory of Pathogen and Biosecurity, Beijing Institute of Microbiology and Epidemiology, Beijing, 100071, China \\ ${ }^{2}$ The First People's Hospital of Lianyungang, Lianyungang, 222002, China \\ ${ }^{3}$ State Key Laboratory for Infectious Disease Prevention and Control, National Institute for Communicable Disease Control \\ and Prevention, Chinese Center for Disease Control and Prevention, Beijing, 102206, China \\ ${ }^{4}$ Collaborative Innovation Center for Diagnosis and Treatment of Infectious Diseases, Hangzhou, 310003, China \\ *These authors contributed equally to this work \\ Correspondence to: Dongsheng Zhou, email: dongshengzhou1977@gmail.com \\ Haijian Zhou, email: zhouhaijian@icdc.cn \\ Jinglin Wang, email: wjlwjl0801@sina.com
}

Keywords: Klebsiella pneumoniae; multidrug resistance; plasmids; p675920-1

Received: July 10, 2017 Accepted: December 05, $2017 \quad$ Published: January 13, 2018

Copyright: Feng et al. This is an open-access article distributed under the terms of the Creative Commons Attribution License 3.0 (CC BY 3.0), which permits unrestricted use, distribution, and reproduction in any medium, provided the original author and source are credited.

\section{ABSTRACT}

This study dealt with detailed genomic characterization of two multidrug resistant (MDR) plasmids p675920-1 and p675920-2 from a single clinical Klebsiella pneumoniae isolate 675920. p675920-1 was essentially a hybrid of the IncFII plasmid pHN7A8 and the IncR plasmid pKPC-LK30, and functioned as an IncFII plasmid with inactivation of the IncR replication gene. The backbone of p675920-2 was a hybrid of a novel replicon, three maintenance regions (22.0-, 2.7-, 2.6-kb in length, respectively) as found in PKPYL2, p10164-3 and pK1HV, respectively, and the entire 25.9-kb conjugal transfer region of pKPYL2. p675920-1 and p675920-2 carried a large number of resistance genes, which contributed to resistance to at least seven classes of antibiotics ( $\beta$-lactams, quinolones, aminoglycosides, fosfomycins, sulphonamides, trimethoprims, and tetracyclines) and one kind of heavy mental (mercury). All of these resistance genes are associated with mobile elements such as insertion sequences, insertion sequence-based transposition units, and transposons, which constituted a total of three novel MDR regions, two in p675920-1 and another in p675920-2. Coexistence of two MDR plasmids p675920-1 and p675920-2 made $K$. pneumoniae 675920 tend to become extensively drug-resistant.

\section{INTRODUCTION}

Plasmids of the incompatibility group IncFII are usually low copy number plasmids ( $>100 \mathrm{~kb}$ in size) with a narrow host range and circulated mainly among Enterobacteriaceae species [1]. Most of IncFII plasmids contain multiple replicons, which are composed of the master IncFII replicon together with one or more other replicons such as IncFIA (replA) [2], IncFIB (replB) [3], and that $(r e p B)$ belonging to an uncharacterized incompatibility group [4]; by contrast, there are some IncFII plasmids, such as R100 (accession number AP000342)
[5] and R1 [6], which contain the sole IncFII replicon. IncFII plasmids with multiple replicons can overcome the incompatibility barrier with incoming plasmids [1]. The IncFII replicon is composed of a four-replication-gene array repA2-6-1-4: repA1 is the core gene for replication initiation, and its expression is primarily dependent upon translation of leader peptide RepA6 and further regulated by the negative regulator RepA2 through inhibition of RepA6 translation [6]; the repA4 region contains the ter (replication terminus) sites for replication termination, and it is also important for stable maintenance of plasmids [7]. IncFII plasmids have been found to carry various antibiotic genes 
and play a key role in dissemination of antibiotic resistance across Enterobacteriaceae species [1].

pHN7A8 (accession number JN232517) is an IncFII single-replicon plasmid from $K$. pneumoniae and has the core IncFII backbone regions for plasmid replication initiation (repA2-6-1-4), maintenance (tir, pemIK, stbAB, $p s i A B$, etc), and conjugal transfer (tra, trb, etc) [8]. All of resistance genes $\left(b l a_{\text {СTX-M- } 65}, b l a_{\text {TEM-1 }}, r m t B\right.$, and fos $\left.A 3\right)$ of this plasmid are harbored within a 14.5-kb MDR region, which is the sole accessory module of pHN7A8 and inserted at a site downstream of pemIK. These resistance genes are associated with mobile elements including one copy of Tn2, IS1, ISEcp1, IS1294, and IS903D and four copies of IS26, which denotes a complex history of transposition and homologous recombination.

The IncR replicon was initially discovered in 2009, and often coexists as an auxiliary replicon with other replicons such as IncA/C, IncF, and IncH [9], constituting multi-replicon plasmids. The IncR replicon alone can promote plasmid replication, and IncR plasmids are herein designated as those containing the sole IncR replicon. The core backbone regions of IncR plasmids are composed of genes or gene loci responsible for plasmid replication initiation $(r e p B)$ and maintenance ( $p a r A B, u m u C D, \operatorname{vag} C D$, and $\operatorname{res} D$ ) but lack conjugal transfer regions, and thus IncR plasmids are not self-transmissible [10]. An array of IncR plasmids, such as pEFER (IncR reference plasmid, accession number CU928144), pKPS30 (accession number KF793937) [9], pKPS77 (accession number KF954150) [9] pKP1780 (accession number JX424614) [11] and pKPCLK30 (accession number KC405622) [12], have been fully sequenced. IncR plasmids have been increasingly reported in Enterobacteriaceae species, and carry various kinds of antibiotic resistance genes.

pKPC-LK30 (accession number KC405622) [12] from $K$. pneumoniae is an untypical IncR plasmid because its backbone contains IncFII-type maintenance regions (carrying par $A B$, relEB, $s t b A B, p s i A B$, uтu $C D$, and $\operatorname{ard} A B$ ) beside the core IncR backbone regions. pKPC-LK30 has a total of two accessory modules, one is a truncated IS 26 element and another is called the $b l a_{\mathrm{KPC}-2}$ region (see below) carrying two resistance genes $b l a_{\mathrm{KPC}-2}$ and $b l a_{\mathrm{SHV}-11}$.

This study dealt with detailed genomic characterization of two novel MDR plasmids p6759201 and p675920-2 coexisting in a clinical K. pneumoniae isolate. These two plasmids displayed complex chimera structures, and harbored not only the backbone regions but the accessory modules composed of a large number of resistance markers and mobile elements.

\section{RESULTS AND DISCUSSION}

\section{Coexistence of p675920-1 and p675920-2 in $K$. pneumoniae}

The multilocus sequence typing (MSLT) showed that the 675920 isolate belonged to the K. pneumoniae sequence type 11 (ST11), with an allelic profile 3-31-1-1-1-4 corresponding to the seven housekeeping gens gapA, infB, $m d h$, pgi, phoE, rpoB, and tonB. PCR screening indicated the presence of $b l a_{\mathrm{KPC}-2}$, but none of the other carbapenemase genes were found in the 675920 isolate.

High-throughput sequencing with genomic DNA of the 675920 isolate generated the circularly closed sequences of two plasmids p675920-1 and p675920-2, which were $164.0 \mathrm{~kb}$ and $79.4 \mathrm{~kb}$ in length, with average $\mathrm{G}+\mathrm{C}$ contents of $53.6 \%$ and $54.1 \%$, and contained 216 and 83 predicted open reading frames (ORFs) in total, respectively (Supplementary Table 1). The modular structure of each plasmid was divided into the backbone regions and the separate accessory modules, which were defined as acquired DNA regions associated with and bordered by mobile elements and inserted at different sites of the backbone (Figure 1, Supplementary Figure 1 and Supplementary Table 1). A total of ten non-redundant genes or gene loci $\left(b l a_{\mathrm{KPC}-2}, b l a_{\mathrm{CTX}-\mathrm{M}-65}, b l a_{\mathrm{TEM}-1}, b l a_{\mathrm{LAP}-2}\right.$, qnrS1, rmtB, tetA(A), sul2, fos A3, and the mer locus), which were involved in resistance to antimicrobials and heavy metal, were found in the accessory modules of these two plasmids (Supplementary Table 1 and Supplementary Table 2).

p675920-2, but not p675920-1, could be transferred into Escherichia coli EC600 through conjugation, generating the transconjugant 675920-tetA-EC600. p675920-1 could be transferred into E. coli TOP10 through electroporation, yielding the electroporant 675920-KPC-TOP10. Class A carbapenemase activities were detected in 675920 and 675920-KPC-TOP10 rather than 675920-tetA-EC600, which were due to production of KPC-2 in the corresponding strains.

The 675920 isolate was resistant to ampicillin, ceftazidime, meropenem, aztreonam, amikacin, tetracycline, ciprofloxacin, azithromycin, trimethoprim, sulfamethoxazole, and nitrofurantoin, but remained susceptible to tigecycline and colistin (Supplementary Table 3). 675920-KPCTOP10 was resistant to the first six drugs but susceptible to all the other tested. 675920-tetA-EC600 was resistant to tetracycline, trimethoprim and sulfamethoxazole, and intermediately resistant to ampicillin and ciprofloxacin, but remained susceptible to all the other drugs tested.

\section{Modular comparison of $\mathrm{p} 675920-1$ with $\mathrm{pCT}$ - KPC, pHN7A8, and pKPC-LK30}

p675920-1 and the IncFII plasmid pCT-KPC (accession number KT185451) from $K$. pneumoniae constituted a novel group of plasmids with highly similar backbones (86\% BLAST query coverage and 99\% maximum nucleotide identity; last accessed on October 24, 2016). The backbones of p675920-1 and pCT-KPC had the identical replication initiation genes (see below), and displayed only two major modular differences: i) a 
15.2-kb backbone region (from $\operatorname{orf375}$ to $\operatorname{par} A$ ) was not found in $\mathrm{pCT}-\mathrm{KPC}$, and ii) the conjugal transfer gene $\Delta$ traI-5> was truncated in pCT-KPC (Supplementary Figure 2). The accessory modules of p675920-1 were composed of the MDR region, the $b l a_{\mathrm{KPC}-2}$ region, the Tn6346-associated region, and IS26, which were highly similar to pCT-KPC with the exception that the 13.6-

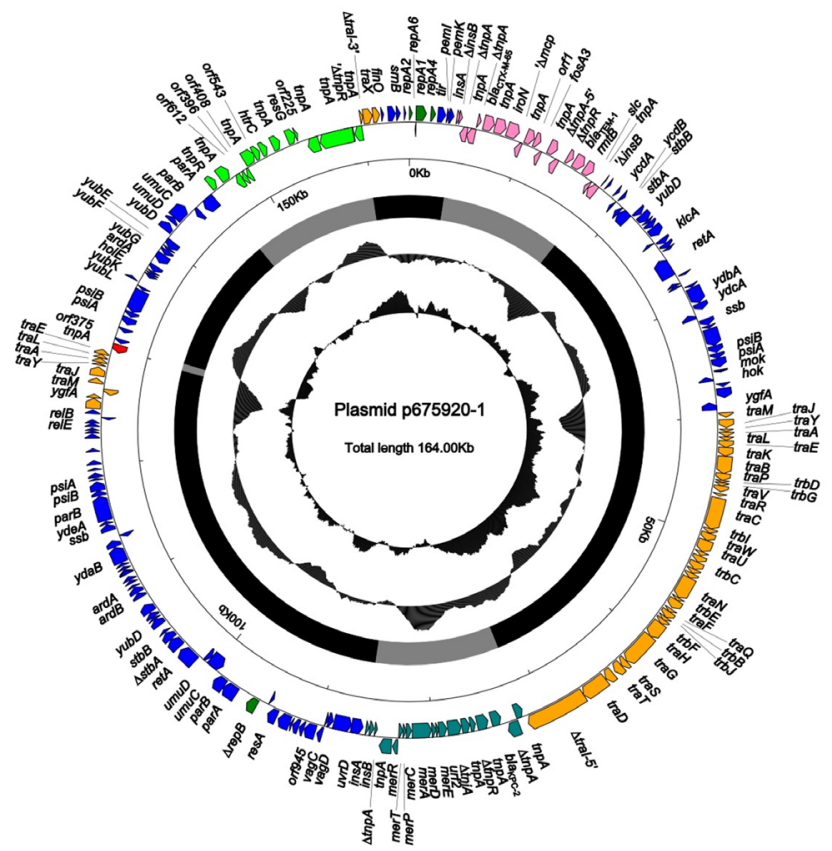

$\mathrm{kb}$ MDR region of $\mathrm{p} 675920$-1 split into two separate regions (a 1.9-kb Tn2-related region and a 14.3-kb MDR region) inserted downstream of pemK and $\Delta r e t A-5$, respectively, in the backbone of pCT-KPC.

p675920-1 and pCT-KPC were the hybrids of a $75.9-\mathrm{kb}$ region derived from $\mathrm{pHN} 7 \mathrm{~A} 8$ [8], an 80.3-kb region derived from pKPC-LK30 [12], and

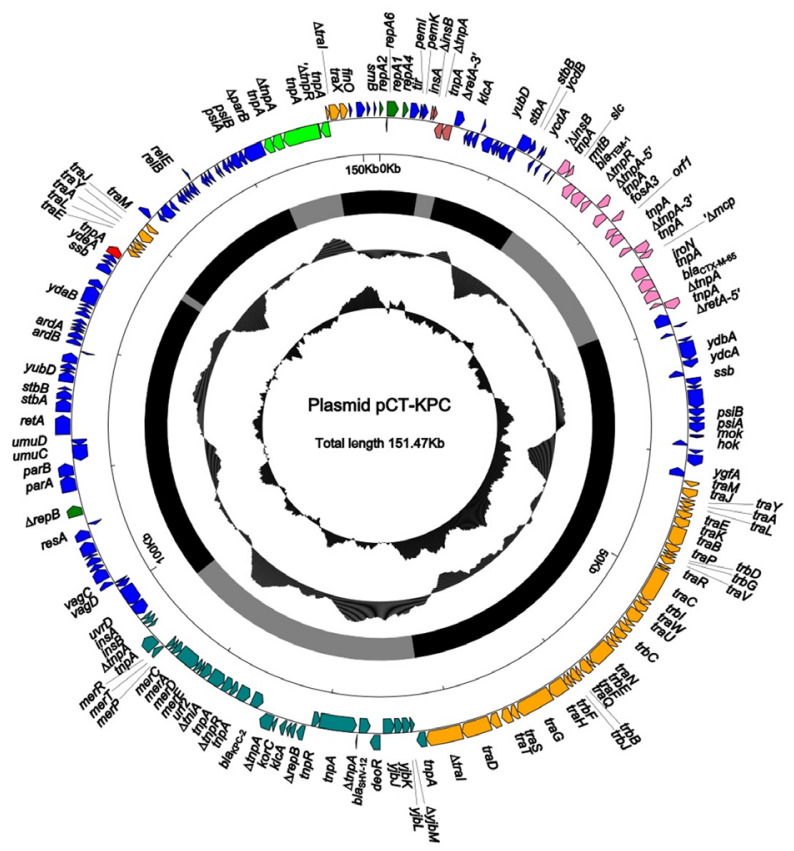

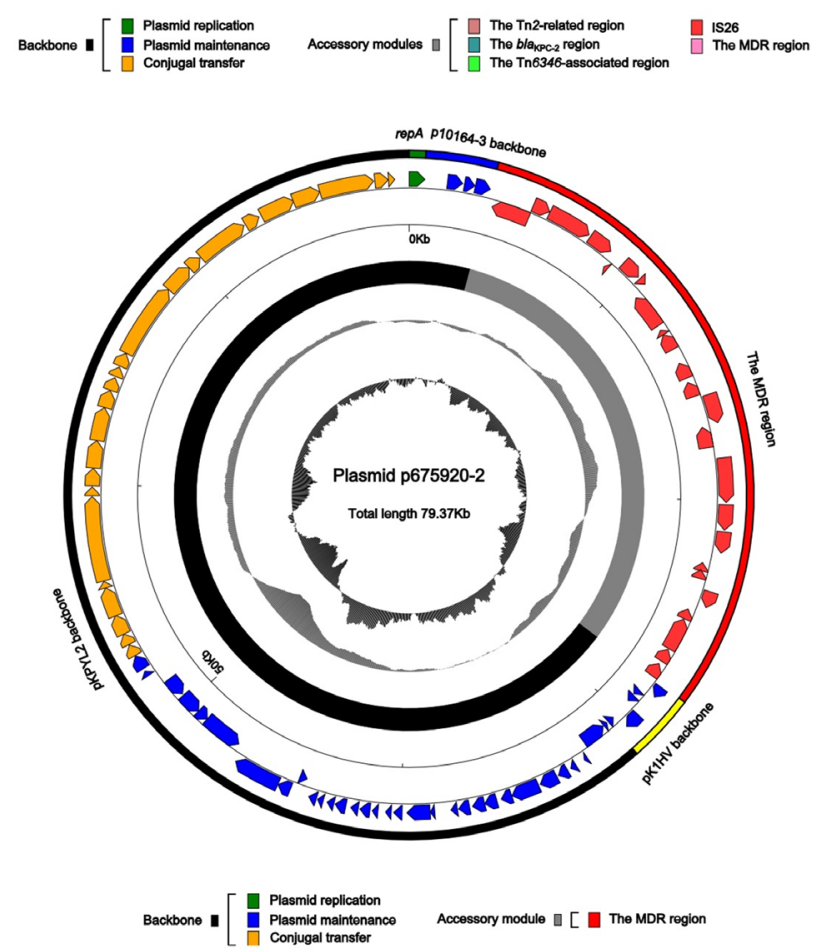

Figure 1: Schematic maps of p675920-1, pCT-KPC, and p675920-2. Genes are denoted by arrows, and the backbone and accessory module regions are highlighted in black and grey, respectively. The innermost circle presents $\mathrm{GC}-\mathrm{skew}[(\mathrm{G}-\mathrm{C}) /(\mathrm{G}+\mathrm{C})]$, with a window size of $500 \mathrm{bp}$ and a step size of $20 \mathrm{bp}$. The next-to-innermost circle presents GC content. 
a 7.0-kb $\Delta$ GIsul2- $\Delta$ Tn6346 region not found in both pHN7A8 and pKPC-LK30 (Supplementary Figure 2). First, the pHN7A8-derived region of p675920-1 differed modularly from the entire pHN7A8 sequence by truncation of IS1294 (Supplementary Figure 2). Second, the pKPC-LK30-derived region of p6759201 was composed of the whole pKPC-LK30 backbone, an intact IS26 element (but the counterpart in pKPCLK30 was truncated), and the $b l a_{\mathrm{KPC}-2}$ region and the Tn6346-associated region (these two accessory regions were originated but slightly differed from the $b l a_{\mathrm{KPC}-2}$ region of pKPC-LK30; see below). In addition, the rep $B$ gene of p675920-1 was pseudogenized and would not participate in plasmid replication initiation. The pKPCLK30-derived region was inserted within the traI gene of the pHN7A8-derived region, which might result in p675920-1 being nonconjugative (Supplementary Figure 2). Third, p675920-1 still contained a 4.9-kb conjugal transfer region as observed in the IncFII $_{K}$ plasmid pKPHS2 (accession number CP003224) [13], which was located within the pKPC-LK30-derived region of p675920-1 but not found in either pKPC-LK30 or pHN7A8 (Supplementary Figure 2).

The hybrid structure of p675920-1 was validated by a set of PCR amplifications that targeted several key jointing fragments (Supplementary Figure 3), using genomic DNA of the 675920 isolate as template.

\section{The MDR regions of pHN7A8, pCT-KPC, and p675920-1}

Two major steps of insertion occurred to eventually assemble the MDR region of pHN7A8 (Supplementary Figures 4 and 2): insertion of the Tn2-rmtB element at a site within ins $B$ of IS $1 R$ (making interruption and further 214-bp truncation of ins $B$ ), and that of a 9.4-kb region (organized in order of IS26, $\triangle \mathrm{Tn} 6377$, the IS26-fos A3IS26 unit [14], and IS1294) at a site between tnp $A$ and tnpR of Tn2 (making truncation of both tnp $A$ and tnpR, and loss of res). The $r m t B$ (aminoglycoside resistance)carrying $\operatorname{Tn} 2-r m t B$ element [15] and its variants [16] were widely found in resistance plasmids from Enterobacteriaceae species. Tn6377 was initially found in pKP96 from K. pneumoniae [17], and generated from insertion of the ISEcp 1-bla $a_{\text {СТХ-M-65 }}-$ IS903D unit [18] at a site within the methyl-accepting chemotaxis gene $m c p$ of the Tn3-family unit transposon Tn1722 [19]. In pHN7A8, Tn6377 was truncated at both ends due to connection of two flanking IS 26 elements, generating

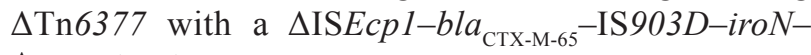
$\Delta m c p$ structure.

The MDR regions from pCT-KPC and p6759201 were genetically closely related to that of pHN7A8 (Figure 2). Compared to the MDR region of $\mathrm{pHN7A8,}$ there were three major modular differences in the counterparts of pCT-KPC: i) the MDR region of pHN7A8 split into two separate regions, namely the Tn2-related region and the MDR region, in pCT-KPC; ii) IS1294 was segmented into two parts $\triangle \mathrm{IS} 1294-5$, and $\triangle \mathrm{IS} 1294-3^{\prime}$ in pCT-KPC, which was followed by inversion of IS26-fos A3-IS26- $\mathrm{IS} 1294-5$ ', and iii) an IS26 element was inserted at a site between $\Delta \operatorname{Tn} 6377$ and $\triangle \mathrm{IS} 1294-5^{\prime}$ in pCT-KPC. The above segmentation and inversion occurred also in the MDR region of p675920-1, but there was further deletion of $\operatorname{IRL}_{\text {IS }} 26$ (inverted repeat left)- $\Delta \mathrm{IS} 1294-5$, , generating a truncated IS26-fos A3-IS26 unit.

\section{The bla $a_{\mathrm{KPC}-2}$ regions of pKPC-LK30, pCT-KPC and p675920-1}

The modular structures of the $b l a_{\mathrm{KPC}-2}$ region of pKPC-LK30 could be divided sequentially as a Tn3family transposon remnant, a truncated IS26-bla $a_{\mathrm{SHV}-12}-$ IS26 unit, $\Delta \operatorname{Tn} 6296-1$, and a Tn21-related region (Figure $3)$. The Tn3-family transposon remnant contained a typical 38-bp IRL element, a truncated core transposition module tnpAR, orf612-orf396-orf408 and orf543 encoding hypothetic proteins, $h \operatorname{tr} C$ (heat shock protein), and ISKpn 26 inserted at a site between orf408 and orf543. Tn6296 was originally identified in the MDR plasmid pKP048 from K. pneumoniae [20], and generated from insertion of the core $b l a_{\mathrm{KPC}-2}$ genetic platform (Tn6376-

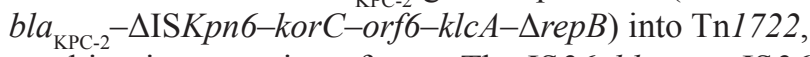
resulting in truncation of $m c p$. The IS26-bla $a_{\mathrm{SHV}-12}-\mathrm{IS} 26$ transposition unit had a structure IS $26-b l a_{\mathrm{SHV}-12}$-the $y g b$ operon-IS26, and its mobilization was catalyzed by the two flanking copies of IS26 [21]. In pKPC-LK30, the 3 '-terminal $\Delta m c p$-Tn6376 region of Tn6296 was lost to form $\Delta \operatorname{Tn} 6296-1$, and the connection of $\Delta \operatorname{Tn} 6296-1$ with the IS $26-b l a_{\mathrm{SHV}-12}-\mathrm{IS} 26$ unit resulted in truncation of the 5'-end IS26. The Tn21-related region in pKPC-LK30 was organized sequentially as a $7.3-\mathrm{kb}$ mer (mercuric resistance)-carrying $\Delta \operatorname{Tn} 21$ region with insertion of IS5075 into the inverted repeat right (IRR), a 288-bp IS903B remnant, and ISKpn14 [22].

The bla $a_{\mathrm{KPC}-2}$ regions of p675920-1, pCT-KPC and pKPC-LK30 were genetically closely related to each other (Figure 3). The loss of the Tn3-family transposon remnant accounted for the sole major modular change in the $b l a_{\mathrm{KPC}-2}$ region of $\mathrm{pCT}-\mathrm{KPC}$ relative to pKPC-LK30. Compared to pKPC-LK30, there were three major modular changes in the bla $a_{\mathrm{KPC}-2}$ region of p675920-1: i) the truncated IS26-bla $a_{\mathrm{SHV}-12}-\mathrm{IS} 26$ unit was entirely deleted, ii) the 5'-terminal region of $\Delta \operatorname{Tn} 6296-1$ was replaced by an IS 26 element, which generated a further truncated structure designated $\Delta \operatorname{Tn} 6296-2$; and iii) the Tn3-family transposon remnant was moved into another accessory module named the Tn6346-associated region (see below). 


\section{The Tn6346-associated regions of p675920-1 and pCT-KPC}

The Tn6346-associated region of p675920-1 was organized in order of the Tn3-family transposon remnant (see above), IS26, GIsul2 remnant, $\Delta \operatorname{Tn} 6346$, and IS26 (Figure 3). Tn6346 was a Tn3-family transposon with a structure IRL-tnpAR-res-mer-IRR [23]. The mercarrying 3'-region of Tn 6346 was replaced by an IS26 element, which left a 1.4-kb remnant composed of the

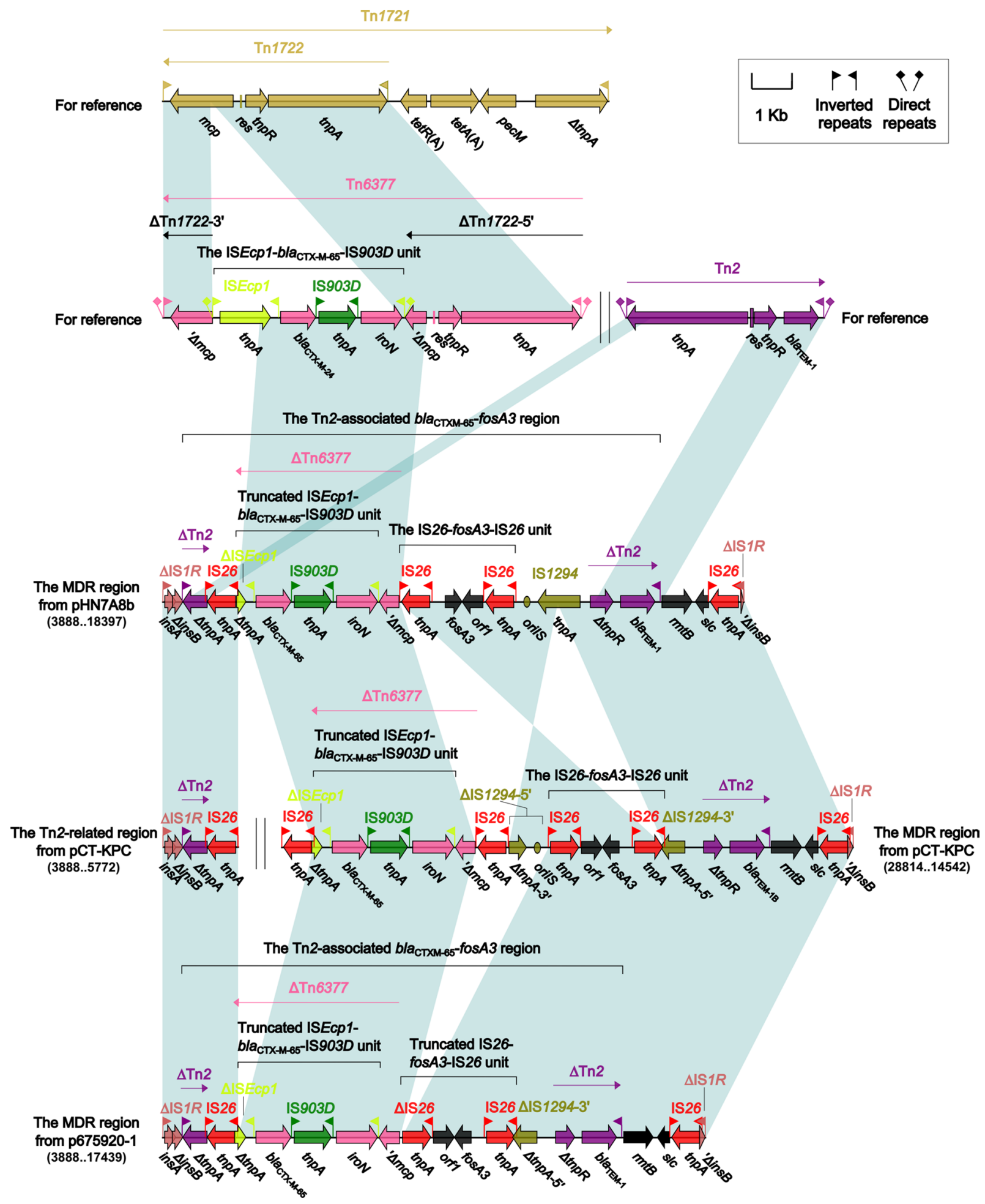

Figure 2: The MDR regions from pHN7A8, pCT-KPC and p675920-1, and comparison with related regions. Genes are denoted by arrows. Genes, mobile elements and other features are colored based on function classification. Shading denotes regions of homology ( $>95 \%$ nucleotide identity). Numbers in brackets indicate the nucleotide positions within the corresponding plasmids. 
IS5075-disrupted IRL (IRL ${ }_{\text {Tn }}$ 6346:IS5075), tnp $A$, and ' $\triangle$ tnpR in p675920-1. GIsul2 is a large integrative and mobile element carrying int (integrase), the resolvase gene $\operatorname{res} G$, several conjugation transfer genes, the sulphonamide resistance gene sul2 and the ISCR2 element, as found in various bacterial species [24]. The GIsul2 remnant was located between the Tn3-family transposon remnant and $\Delta \operatorname{Tn} 6346$ in p675920-1 and composed of only res $G$ and an unknown function gene orf 225. In the Tn6346-associated region of pCT-KPC, an IS26 element (instead of the fragment composed of the Tn3-family transposon remnant, IS26 and the GIsul2 remnant as observed in p675920-1) was connected with $\Delta \operatorname{Tn} 6346$, leading to truncation of $\mathrm{IRL}_{\mathrm{Tn}} 6346: \mathrm{IS} 5075$ (Figure 3). The Tn6346-associated regions from both p675920-1 and pCT-KPC carried none of resistance genes.

\section{Modular comparison of p675920-2 with pKPYL2}

The whole sequence of p675920-2 was mostly similar to pKPYL2 (accession number CP013340) from Raoultella ornithinolytica, with $68 \%$ query coverage and 98\% nucleotide identity (last accessed October 24, 2016). pKPYL2 and p675920-2 contained two different replication initiation rep $A$ genes with extremely low identity, both of which could not be assigned into any of known incompatibility groups. The entire conjugal transfer region of p675920-2 was essentially identical to pKPYL2. The plasmid maintenance regions of p6759202 could be divided into three separate parts (48.8-, 2.7-, 2.6-kb in length, respectively), which were also observed in pKPYL2, p10164-3 (accession number KX710094) [25] and pK1HV (accession number HF545434) [26], respectively (Figure 1). pKPYL2 contained a total of 12 accessory modules, associated with Tn5403, a Tn3family transposon remnant and various IS elements, but carried none of resistance genes. p675920-2 harbored a single accessory module, namely the $24.7-\mathrm{kb}$ MDR region, which was inserted at a site between p10164-3 and pK1HV derived maintenance regions.

\section{The MDR region of $\mathrm{p} 675920-2$}

The MDR region of p675920-2 could be divided into the Tn1721- $\Delta$ GIsul2 region and the truncated

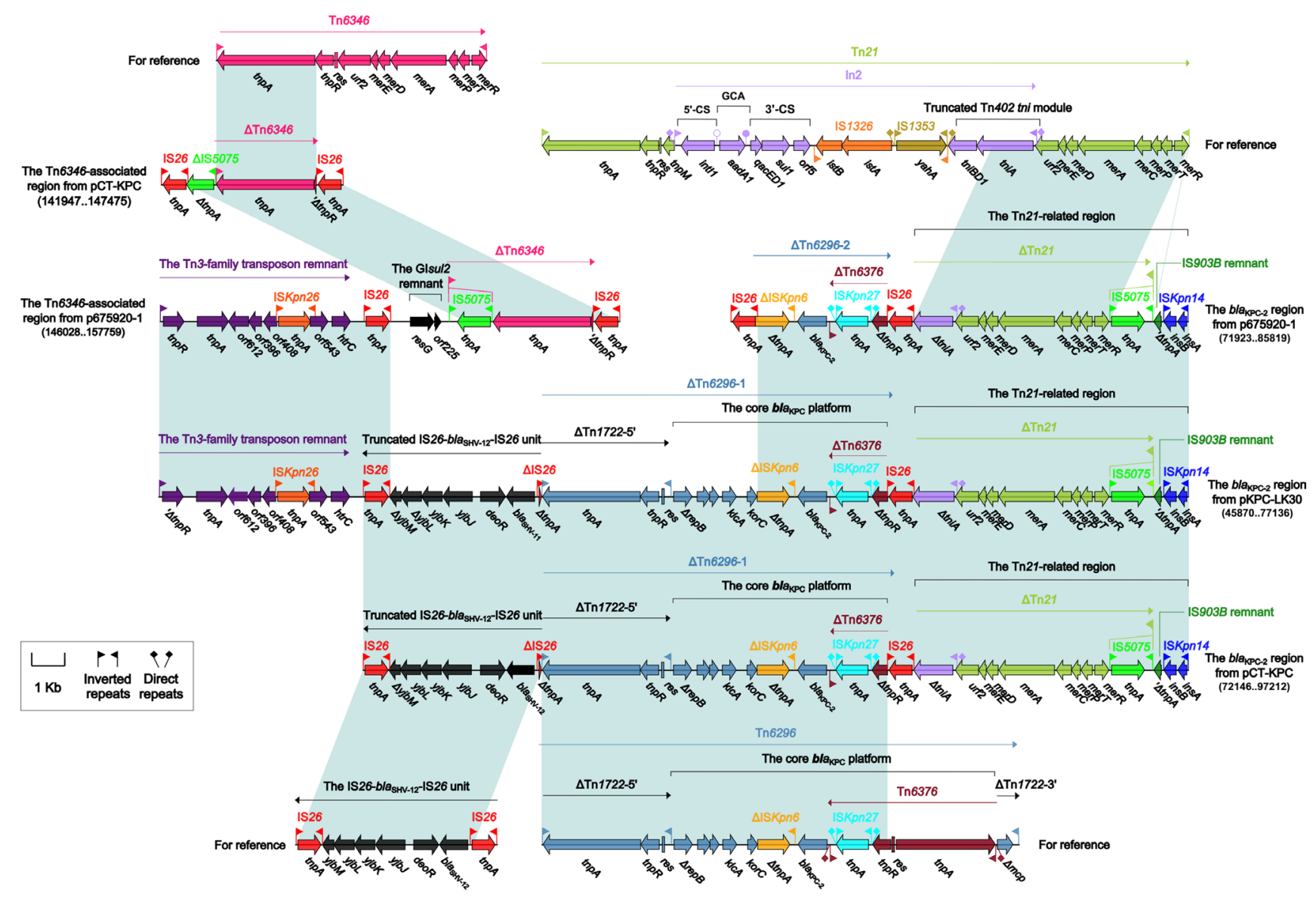

Figure 3: The blaKPC-2 regions from pKPC-LK30, pCT-KPC and p675920-1, the Tn6346-associated regions from p675920-1 and pCT-KPC, and comparison with related regions. Genes are denoted by arrows. Genes, mobile elements and other features are colored based on function classification. Shading denotes regions of homology ( $>95 \%$ nucleotide identity). Numbers in brackets indicate the nucleotide positions within the corresponding plasmids. 
IS26-bla LAP-2 $_{\text {- } q n r S 1-I S 26}$ unit (Figure 4). The Tn1721$\triangle \mathrm{GIsul2}$ region was generated from insertion of the IS26- $\Delta$ Tn6346- $\Delta$ GIsul2-IS26 unit (flanked by two oppositely oriented IS26 elements) into Tn1721 [19] containing the class $\mathrm{A}$ tetracycline resistance module $\operatorname{tet} A(\mathrm{~A})-\operatorname{tet} R(\mathrm{~A})$, which led to truncation of $m c p$ as well as loss of res-tnpRA. $\Delta$ GIsul2 was a 3'-terminal region of GIsul2 with a resG-orf225-ISCR2-glmM-sul2 structure.

The IS26-bla $a_{\text {LAP-2 }}-q n r S 1-I S 26$ unit, as observed in pE66An from E. coli [26], had two flanking copies of IS26 in direct orientation. This unit contained the core qnrS1 (quinolone resistance) region displaying an $\triangle \mathrm{ISECl2}$ $q n r S 1-\Delta t n p R-I S K p n 19$ structure, which was widely found in qnrS1-carrying plasmids from Enterobacteriaceae species [25]. The upstream ISEcl2 element could play an important role in original acquisition of qnrS1 [27], while ISKpn19 might contribute to mobilization of the core $q n r S 1$ region. The $b l a_{\mathrm{LAP}-2}$ gene was located upstream of the core $q n r S 1$ region and conferred resistance to narrowspectrum $\beta$-lactams. In p675920-1, IS26- $\Delta$ ftsI-5' and IS26 were absent at $5^{\prime}-$ and $3^{\prime}$-ends of the IS $26-b l a_{\text {LAP-2 }}$ qnrS1-IS26 unit, respectively.

\section{MATERIALS AND METHODS}

\section{Bacterial strain and identification}

The use of human specimens and all related experimental protocols were approved by the Committee on Human Research of the First People's Hospital of Lianyungang, and carried out in accordance with the approved guidelines. The research involving biohazards and all related procedures were approved by the Biosafety Committee of the Beijing Institute of Microbiology and Epidemiology. K. pneumoniae isolate 675920 was isolated in 2015 from a sputum specimen from an elderly male with pulmonary infection in a public hospital in Lianyungang City, China. Bacterial species identification was performed by $16 \mathrm{~S}$ rDNA gene sequencing [28] and by PCR detection of $K$. pneumoniae-specific khe gene [29]. The MLST scheme for K. pneumoniae was derived from the PasteurMLST database (http://bigsdb.pasteur. fr/klebsiella/). The major plasmid-borne carbapenemase and ESBL genes were screened for by PCR [30]. All PCR amplicons were sequenced on ABI 3730 Sequencer (LifeTechnologies, CA, USA) using the PCR primers.

\section{Sequencing and annotation}

Genomic DNA was isolated from the 675920 isolate using a Qiagen large construct kit, and sequenced from a mate-pair library with average insert size of 5,000 bp, using a MiSeq sequencer (Illumina, CA, USA). Sequence assembly and annotation were performed as described previously [31]. Briefly, the contigs were assembled using Newbler 2.6 [32]. Open reading frames and pseudogenes were predicted using RAST 2.0 [33] combined with BLASTP/BLASTN searches [34] against the UniProtKB/Swiss-Prot database [35] and the RefSeq
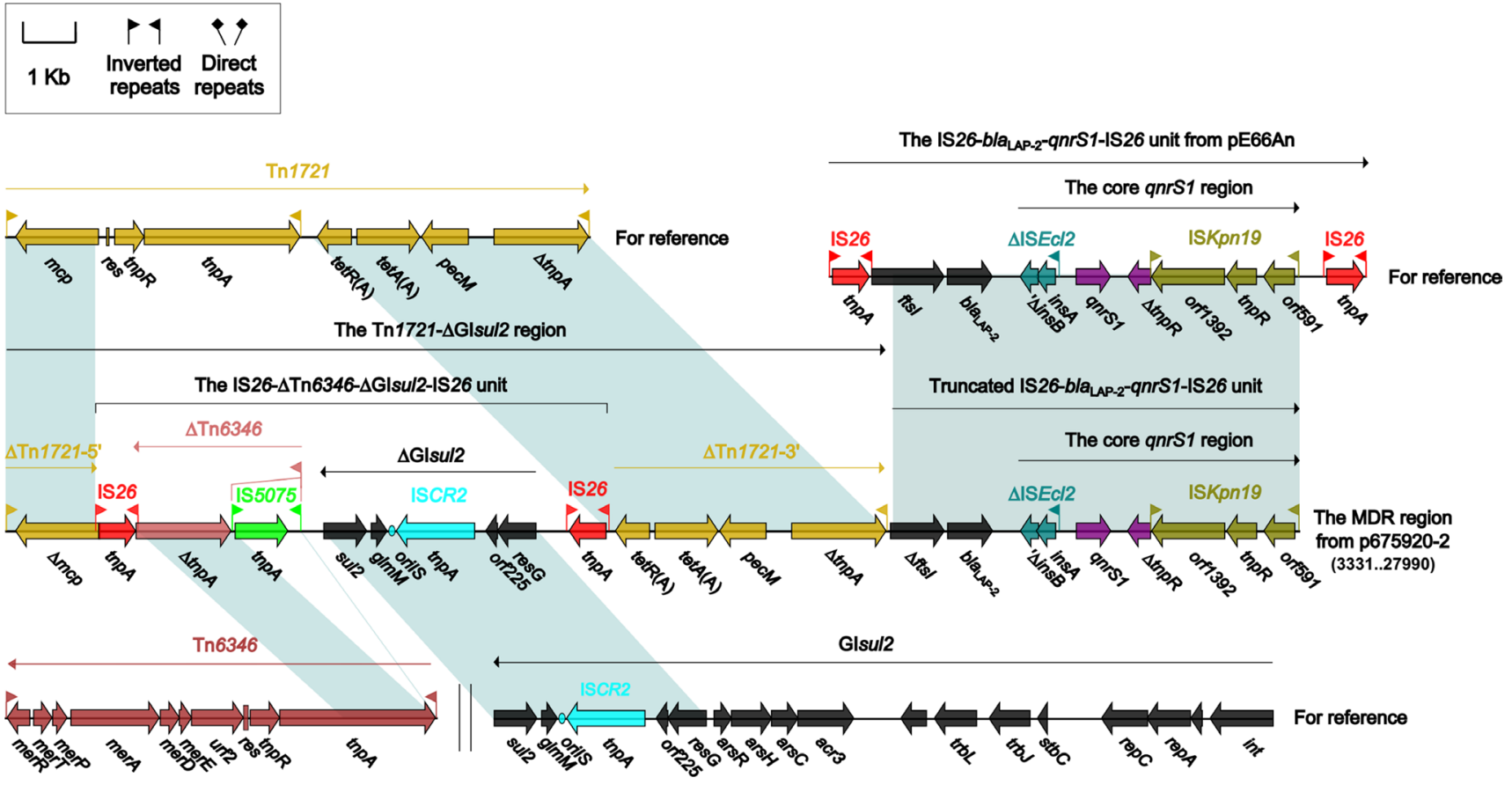

Figure 4: The MDR region from p675920-2, and comparison with related regions. Genes are denoted by arrows. Genes, mobile elements and other features are colored based on function classification. Shading denotes regions of homology $(>95 \%$ nucleotide identity). Numbers in brackets indicate the nucleotide positions within p675920-2. 
database [36]. Annotation of resistance genes, mobile elements, and other features was carried out using the online databases including CARD [37], ResFinder [38], BacMet [39], ISfinder [40], INTEGRALL [41], and the Tn Number Registry [42]. Multiple and pairwise sequence comparisons were performed using MUSCLE 3.8.31 [43] and BLASTN, respectively. Gene organization diagrams were drawn in Inkscape 0.48.1.

\section{Plasmid transfer}

Plasmids were transferred in attempt from the 675920 isolate into E. coli TOP10 and EC600 (highly resistant to rifampicin) through electroporation and conjugal transfer, respectively [31]. For selection of electroporant or transconjugant containing the tet $A(\mathrm{~A})$ or $b l a_{\mathrm{KPC}}$ marker, $10 \mu \mathrm{g}$ / $\mathrm{ml}$ tetracycline, $2 \mu \mathrm{g} / \mathrm{ml}$ imipenem, and $1000 \mu \mathrm{g} / \mathrm{ml}$ rifampicin were used in accordance with specific circumstances.

\section{Phenotypic assays}

Activity of Ambler class A/B/D carbapenemases in bacterial cell extracts was determined by a modified CarbaNP test [31]. Bacterial antimicrobial susceptibility was tested by the broth dilution method, and interpreted as per CLSI guidelines [44].

\section{Nucleotide sequence accession numbers}

The p675920-1 and p675920-2 sequences were submitted to GenBank under accession numbers MF133495 and MF133496, respectively.

\section{CONCLUSIONS}

The two coexisting MDR plasmids p675920-1 and p675920-2 display complex chimera structures. p6759201 is essentially a hybrid of the IncFII single-replicon plasmid pHN7A8 and the IncR plasmid pKPC-LK30, and almost all of the backbone and accessory regions of pKPC-LK30 and pHN7A8 are presented in p675920-1. p675920-1 still contains two additional regions, i.e., a 4.9-kb conjugal transfer region and a 7.0-kb $\Delta$ GIsul2$\Delta \mathrm{Tn} 6346$ region, which are not presented in both pKPCLK30 and pHN7A8. p675920-1 is not self-transmissible because the conjugal transfer region originated from pHN7A8 is interrupted by insertion of the pKPC-LK30derived region. p675920-1 functions as an IncFII plasmid because the IncR replicon is inactivated.

p675920-1 contains a total of four accessory modules inserted at different sites across the backbone, and among them are two large resistance regions, which harbor a lot of resistance genes or gene loci (such as bla $a_{\mathrm{KPC}-2}, r m t B$, fos $A 3$, and $m e r$ ) and associated mobile elements (such as insertion sequences, insertion sequence-based transposition units, and transposons). A total of six copies of IS26 are presented in the two large resistance regions of p6759201. All of the regions flanked by two IS26 elements lack paired short direct repeats (DRs; target site duplication signals for transposition) at both ends and therefore cannot be annotated as typical IS26-composite transposons. The common component IS26 would act as an adaptor to mediate massive recombination and transposition events [45-47] and thereby contribute to the assembly of these two large resistance regions in p675920-1.

Whilst the preparation of this manuscript, plasmid pKp_Goe_414-4 (accession number CP018341) from $K$. pneumoniae, which shows $98 \%$ query coverage and $99 \%$ nucleotide identity to p675920-2, was released in GenBank. p675920-2 and pKp_Goe_414-4 display very low levels of homology to available sequences in public databases and, thus, represent a novel group of plasmids. The backbone of p675920-2 displays a mosaic structure composed of a novel replicon, three maintenance regions as found in pKPYL2, p10164-3 and pK1HV, respectively, and the entire conjugal transfer region of pKPYL2. This novel replicon is able to coordinate with the maintenance and conjugal transfer regions originated from various plasmids, and maintain stable replication of pKPYL2 at a steadystate copy number. In addition, p675920-2 has evolved to integrate a sole accessory module that carries multiple resistance determinants such as qnrS1, tetA(A), sul2, and $b l a_{\mathrm{LAP}-2} \cdot \mathrm{p} 675920-2$ is self-transmissible because it contains the intact conjugal transfer region originated from pKPYL2.

Coexistence of p675920-1 and p675920-2 makes K. pneumoniae 675920 tend to become extensively drugresistant, thus resulting in limited choice of antibiotics for treatment and increased risks of death. Surveillance and epidemiological studies are needed to evaluate prevalence of p675920-1- and p675920-2-like plasmids among $K$. pneumoniae isolates.

\section{Abbreviations}

MDR: multidrug resistance; ESBL: extendedspectrum $\beta$-lactamase; ORFs: open reading frames; IS: insertion sequence; IRL: inverted repeat left; IRR: inverted repeat right; DRs: direct repeats.

\section{Author contributions}

DS.Z., HJ.Z. and JL.W. conceived the study and designed experimental procedures. J.F., Z.Y., Z.Z. and HF.M. performed the experiments. J.F., DS.Z., and Y.Z. analyzed the data. XY.J., LJ.Z., WH.Y and HY.Y. contributed to reagents and materials. DS.Z., J.F., HJ.Z., and JL.W. wrote this manuscript.

\section{CONFLICTS OF INTEREST}

All authors declare that there are no conflicts of interest. 


\section{FUNDING}

This work was supported by AWS15J006, the Special Key Project of Biosafety Technologies (2016YFC1202600) for the National Major Research \& Development Program of China, and the Foundation of the State Key Laboratory of Pathogen and Biosecurity of China (SKLPBS1417).

\section{REFERENCES}

1. Villa L, Garcia-Fernandez A, Fortini D, Carattoli A. Replicon sequence typing of IncF plasmids carrying virulence and resistance determinants. J Antimicrob Chemother. 2010; 65:2518-29. https://doi.org/10.1093/jac/dkq347.

2. Woodford N, Carattoli A, Karisik E, Underwood A, Ellington MJ, Livermore DM. Complete nucleotide sequences of plasmids pEK204, pEK499, and pEK516, encoding CTX-M enzymes in three major Escherichia coli lineages from the United Kingdom, all belonging to the international O25:H4-ST131 clone. Antimicrob Agents Chemother. 2009; 53:4472-82. https://doi.org/10.1128/ AAC.00688-09.

3. Fricke WF, Wright MS, Lindell AH, Harkins DM, BakerAustin C, Ravel J, Stepanauskas R. Insights into the environmental resistance gene pool from the genome sequence of the multidrug-resistant environmental isolate Escherichia coli SMS-3-5. J Bacteriol. 2008; 190:6779-94. https://doi.org/10.1128/JB.00661-08.

4. Wang L, Fang H, Feng J, Yin Z, Xie X, Zhu X, Wang J, Chen W, Yang R, Du H, Zhou D. Complete sequences of KPC-2-encoding plasmid p628-KPC and CTX-M-55encoding p628-CTXM coexisted in Klebsiella pneumoniae. Front Microbiol. 2015; 6:838. https://doi.org/10.3389/ fmicb.2015.00838.

5. Ohtsubo H, Ryder TB, Maeda Y, Armstrong K, Ohtsubo E. DNA replication of the resistance plasmid R100 and its control. Advances in Biophysics. 1986; 21:115-33.

6. Blomberg P, Nordström K, Wagner EG. Replication control of plasmid R1: RepA synthesis is regulated by CopA RNA through inhibition of leader peptide translation. The EMBO Journal. 1992; 11:2675-83.

7. Jiang T, Min YN, Liu W, Womble DD, Rownd RH. Insertion and deletion mutations in the repA4 region of the IncFII plasmid NR1 cause unstable inheritance. J Bacteriol. 1993; 175:5350.

8. He L, Partridge SR, Yang X, Hou J, Deng Y, Yao Q, Zeng $\mathrm{Z}$, Chen Z, Liu JH. Complete nucleotide sequence of pHN7A8, an F33:A-:B- type epidemic plasmid carrying blaCTX-M-65, fosA3 and rmtB from China. Journal of Antimicrobial Chemotherapy. 2013; 68:46-50.

9. Compain F, Frangeul L, Drieux L, Verdet C, Brisse S, Arlet G, Decre D. Complete nucleotide sequence of two multidrug-resistant IncR plasmids from Klebsiella pneumoniae. Antimicrob Agents Chemother. 2014; 58:4207-10. https://doi.org/10.1128/AAC.02773-13.
10. Alvarado A, Garcillan-Barcia MP, de la Cruz F. A degenerate primer MOB typing (DPMT) method to classify gamma-proteobacterial plasmids in clinical and environmental settings. Plos One. 2012; 7:e40438. https:// doi.org/10.1371/journal.pone.0040438.

11. Papagiannitsis CC, Miriagou V, Giakkoupi P, Tzouvelekis LS, Vatopoulos AC. Characterization of pKP1780, a novel IncR plasmid from the emerging Klebsiella pneumoniae ST147, encoding the VIM-1 metallo-beta-lactamase. J Antimicrob Chemother. 2013; 68:2259-62. https://doi. org/10.1093/jac/dkt196.

12. Chen YT, Lin JC, Fung CP, Lu PL, Chuang YC, Wu TL, Siu LK. KPC-2-encoding plasmids from Escherichia coli and Klebsiella pneumoniae in Taiwan. J Antimicrob Chemother. 2014; 69:628-31. https://doi.org/10.1093/jac/dkt409.

13. Liu P, Li P, Jiang X, Bi D, Xie Y, Tai C, Deng Z, Rajakumar $\mathrm{K}$, Ou HY. Complete genome sequence of Klebsiella pneumoniae subsp. pneumoniae HS11286, a multidrugresistant strain isolated from human sputum. J Bacteriol. 2012; 194:1841-2. https://doi.org/10.1128/JB.00043-12.

14. Ho PL, Chan J, Lo WU, Law PY, Li Z, Lai EL, Chow KH. Dissemination of plasmid-mediated fosfomycin resistance fosA3 among multidrug-resistant Escherichia coli from livestock and other animals. Journal of Applied Microbiology. 2013; 114:695-702.

15. Doi Y, Adams-Haduch JM, Paterson DL. Escherichia coli isolate coproducing 16S rRNA Methylase and CTXM-type extended-spectrum beta-lactamase isolated from an outpatient in the United States. Antimicrob Agents Chemother. 2008; 52:1204-5. https://doi.org/10.1128/ AAC.01320-07.

16. Martins W, Gales AC. Frequent Tn2 Misannotation in the Genetic Background of rmtB. Antimicrob Agents Chemother. 2017. https://doi.org/10.1128/AAC.00811-17.

17. Shen P, Jiang Y, Zhou Z, Zhang J, Yu Y, Li L. Complete nucleotide sequence of pKP96, a 67850 bp multiresistance plasmid encoding qnrA1, aac(6')-Ib-cr and blaCTX-M-24 from Klebsiella pneumoniae. J Antimicrob Chemother. 2008; 62:1252-6. https://doi.org/10.1093/jac/dkn397.

18. Zong Z, Yu R, Wang X, Lu X. blaCTX-M-65 is carried by a Tn1722-like element on an IncN conjugative plasmid of ST131 Escherichia coli. J Med Microbiol. 2011; 60:435-41. https://doi.org/10.1099/jmm.0.026997-0.

19. Partridge SR. Analysis of antibiotic resistance regions in Gram-negative bacteria. FEMS Microbiol Rev. 2011; 35:820 55. https://doi.org/10.1111/j.1574-6976.2011.00277.x.

20. Jiang Y, Yu D, Wei Z, Shen P, Zhou Z, Yu Y. Complete nucleotide sequence of Klebsiella pneumoniae multidrug resistance plasmid pKP048, carrying blaKPC-2, blaDHA-1, qnrB4, and armA. Antimicrob Agents Chemother. 2010; 54:3967-9. https://doi.org/AAC.00137-10.

21. Ford PJ, Avison MB. Evolutionary mapping of the SHV betalactamase and evidence for two separate IS26-dependent blaSHV mobilization events from the Klebsiella pneumoniae chromosome. J Antimicrob Chemother. 2004; 54:69-75. 
22. Liebert CA, Hall RM, Summers AO. Transposon Tn21, flagship of the floating genome. Microbiol Mol Biol Rev. 1999; 63:507-22.

23. Ng SP, Davis B, Palombo EA, Bhave M. A Tn5051-like mer-containing transposon identified in a heavy metal tolerant strain Achromobacter sp. AO22. BMC Res Notes. 2009; 2:38. https://doi.org/10.1186/1756-0500-2-38.

24. Nigro SJ, Hall RM. GIsul2, a genomic island carrying the sul2 sulphonamide resistance gene and the small mobile element CR2 found in the Enterobacter cloacae subspecies cloacae type strain ATCC 13047 from 1890, Shigella flexneri ATCC 700930 from 1954 and Acinetobacter bauman. J Antimicrob Chemother. 2011; 66:2175-6.

25. Sun F, Zhou D, Sun Q, Luo W, Tong Y, Zhang D, Wang Q, Feng W, Chen W, Fan Y, Xia P. Genetic characterization of two fully sequenced multi-drug resistant plasmids pP10164-2 and pP10164-3 from Leclercia adecarboxylata. Sci Rep. 2016; 6:33982. https://doi.org/10.1038/srep33982.

26. Le V, Nhu NT, Cerdeno-Tarraga A, Campbell JI, Tuyen HT, Nhu Tdo H, Tam PT, Schultsz C, Thwaites G, Thomson NR, Baker S. Genetic characterization of three qnrS1-harbouring multidrug-resistance plasmids and qnrS1-containing transposons circulating in Ho Chi Minh City, Vietnam. J Med Microbiol. 2015; 64:869-78. https://doi.org/10.1099/ jmm.0.000100.

27. Poirel L, Leviandier C, Nordmann P. Prevalence and genetic analysis of plasmid-mediated quinolone resistance determinants QnrA and QnrS in Enterobacteriaceae isolates from a French university hospital. Antimicrob Agents Chemother. 2006; 50:3992-7. https://doi.org/10.1128/AAC.00597-06.

28. Frank JA, Reich CI, Sharma S, Weisbaum JS, Wilson BA, Olsen GJ. Critical evaluation of two primers commonly used for amplification of bacterial 16S rRNA genes. Appl Environ Microbiol. 2008; 74:2461-70. https://doi. org/10.1128/AEM.02272-07.

29. Yin-Ching C, Jer-Horng S, Ching-Nan L, Ming-Chung C. Cloning of a gene encoding a unique haemolysin from Klebsiella pneumoniae and its potential use as a speciesspecific gene probe. Microb Pathog. 2002; 33:1-6.

30. Chen Z, Fang H, Wang L, Sun F, Wang Y, Yin Z, Yang H, Yang W, Wang J, Xia P, Zhou D, Liu C. IMP-1 encoded by a novel Tn402-like class 1 integron in clinical Achromobacter xylosoxidans, China. Sci Rep. 2014; 4:7212. https://doi. org/10.1038/srep07212.

31. Feng W, Zhou D, Wang Q, Luo W, Zhang D, Sun Q, Tong Y, Chen W, Sun F, Xia P. Dissemination of IMP-4-encoding pIMP-HZ1-related plasmids among Klebsiella pneumoniae and Pseudomonas aeruginosa in a Chinese teaching hospital. Sci Rep. 2016; 6:33419. https://doi.org/10.1038/srep33419.

32. Nederbragt AJ. On the middle ground between open source and commercial software - the case of the Newbler program. Genome Biol. 2014; 15:113.

33. Brettin T, Davis JJ, Disz T, Edwards RA, Gerdes S, Olsen GJ, Olson R, Overbeek R, Parrello B, Pusch GD, Shukla M, Thomason JA 3rd, Stevens R, et al. RASTtk: a modular and extensible implementation of the RAST algorithm for building custom annotation pipelines and annotating batches of genomes. Sci Rep. 2015; 5:8365. https://doi. org/10.1038/srep08365.

34. Boratyn GM, Camacho C, Cooper PS, Coulouris G, Fong A, Ma N, Madden TL, Matten WT, McGinnis SD, Merezhuk Y, Raytselis Y, Sayers EW, Tao T, et al. BLAST: a more efficient report with usability improvements. Nucleic Acids Res. 2013; 41:W29-33. https://doi.org/10.1093/nar/gkt282.

35. Boutet E, Lieberherr D, Tognolli M, Schneider M, Bansal P, Bridge AJ, Poux S, Bougueleret L, Xenarios I. UniProtKB/ Swiss-Prot, the Manually Annotated Section of the UniProt KnowledgeBase: How to Use the Entry View. Methods Mol Biol. 2016; 1374:23-54. https://doi.org/10.1007/9781-4939-3167-5_2.

36. O'Leary NA, Wright MW, Brister JR, Ciufo S, Haddad D, McVeigh R, Rajput B, Robbertse B, Smith-White B, AkoAdjei D, Astashyn A, Badretdin A, Bao Y, et al. Reference sequence (RefSeq) database at NCBI: current status, taxonomic expansion, and functional annotation. Nucleic Acids Res. 2016; 44:D733-45. https://doi.org/10.1093/nar/gkv1189.

37. Jia B, Raphenya AR, Alcock B, Waglechner N, Guo P, Tsang KK, Lago BA, Dave BM, Pereira S, Sharma AN, Doshi S, Courtot M, Lo R, et al. CARD 2017: expansion and model-centric curation of the comprehensive antibiotic resistance database. Nucleic Acids Res. 2016. https://doi. org/10.1093/nar/gkw1004.

38. Zankari E, Hasman H, Cosentino S, Vestergaard M, Rasmussen S, Lund O, Aarestrup FM, Larsen MV. Identification of acquired antimicrobial resistance genes. J Antimicrob Chemother. 2012; 67:2640-4. https://doi.org/10.1093/jac/dks261.

39. Pal C, Bengtsson-Palme J, Rensing C, Kristiansson E, Larsson DG. BacMet: antibacterial biocide and metal resistance genes database. Nucleic Acids Res. 2014; 42:D737-43. https://doi.org/10.1093/nar/gkt1252.

40. Siguier P, Perochon J, Lestrade L, Mahillon J, Chandler M. ISfinder: the reference centre for bacterial insertion sequences. Nucleic Acids Res. 2006; 34:D32-6. https://doi. org/10.1093/nar/gkj014.

41. Moura A, Soares M, Pereira C, Leitao N, Henriques I, Correia A. INTEGRALL: a database and search engine for integrons, integrases and gene cassettes. Bioinformatics. 2009; 25:10968. https://doi.org/10.1093/bioinformatics/btp105.

42. Roberts AP, Chandler M, Courvalin P, Guedon G, Mullany P, Pembroke T, Rood J, Smith CJ, Summers AO, Tsuda M, Berg DE. Revised nomenclature for transposable genetic elements. Plasmid. 2008; 60:167-73. https://doi. org/10.1016/j.plasmid.2008.08.001.

43. Edgar RC. MUSCLE: multiple sequence alignment with high accuracy and high throughput. Nucleic Acids Res. 2004; 32:1792-7. https://doi.org/10.1093/nar/gkh340.

44. Clinical and Laboratory Standards Institute. M100-S25 Performance standards for antimicrobial susceptibility testing: twenty-fifth informational supplement. (Wayne, PA, USA: CLSI). http://www.facm.ucl.ac.be/intranet/CLSI/ CLSI-2015-M100-S25-original.pdf. 
45. Harmer CJ, Moran RA, Hall RM. Movement of IS26associated antibiotic resistance genes occurs via a translocatable unit that includes a single IS26 and preferentially inserts adjacent to another IS26. MBio. 2014; 5:e01801-14. https://doi.org/10.1128/mBio.01801-14.

46. He S, Hickman AB, Varani AM, Siguier P, Chandler M, Dekker JP, Dyda F. Insertion Sequence IS26 Reorganizes Plasmids in Clinically Isolated Multidrug-Resistant Bacteria by Replicative Transposition. MBio. 2015; 6:e00762. https://doi.org/10.1128/mBio.00762-15.

47. Harmer CJ, Hall RM. IS26-Mediated Formation of Transposons Carrying Antibiotic Resistance Genes. mSphere. 2016; 1. https://doi.org/10.1128/mSphere.00038-16. 